\title{
Memory for faces: Encoding and retrieval operations
}

\author{
GARY L. WELLS and BRENDA HRYCIW \\ University of Alberta, Edmonton, Alberta, Canada
}

\begin{abstract}
The reliable finding that trait judgments of faces yield better recognition memory than do feature judgments of faces is conceptualized as an encoding-specificity effect. Specifically, both trait-judgment encodings of faces and face-recognition tests are argued to be holistic, involv. ing topographical information with between-feature processing. Consistent with the concept that encoding and retrieval operations interact to produce retrieval success, it was expected that a memory-for-face test using the Identi-kit (which requires reconstructions of the face at a feature level of analysig) would show trait-encoding tasks to be inferior to feature-encoding tasks. Eighty subjects were assigned randomly to judge a face on 10 trait dimensions (e.g., honesty-dishonesty) or on 10 feature dimensions (e.g., narrow nose-wide nose) and subsequently attempted to recognize the target among five distractors or to reconstruct the face from an Identi-kit. The significant interaction between encoding and retrieval operations indicated that the face was best identified under trait-encoding conditions but best reconstructed under featureencoding conditions. The match between trait encoding and recognition in yielding high memory performance suggests strongly that trait judgments foster holistic processing of faces (i.e., interfeature topographical information is part of the context) and that the recognition of faces also is holistic. Finally, the utility of the feature vs. holistic-processing distinction is questioned, and an alternative is proposed.
\end{abstract}

One of the most reliable findings in memory for human faces is that trait judgments of a face yield better subsequent recognition memory than do physical-feature judgments (e.g., Baddeley, 1979; Bower \& Karlin, 1974; Mueller, Carlomusto, \& Goldstein, 1978; Patterson \& Baddeley, 1977; Strnad \& Mueller, 1977; Warrington \& Ackroyd, 1975; Winograd, 1976). The interpretation of this effect has revolved around the levels-of-processing framework (Craik \& Lockhart, 1972), in which the traitjudgment-encoding task is thought to evoke a deeper, more elaborate processing of the face than does the feature-encoding task. Winograd (1981) suggested that trait or similar judgments (e.g., judgments of occupation) produce better memory for faces than do feature judgments because the former increase the number of features processed. Interestingly, trait-judgment-encoding instructions also produce better recognition memory for faces than do instructions to the observer to try to remember the face (Warrington \& Ackroyd, 1975).

Although Winograd (1981) suggested that the critical difference between trait- and feature-encoding operations of faces rests with the number of features, especially distinctive features, little progress has been made in delineating the processes involved. We suggest that the

This research was supported by a grant from the Social Sciences and Humanities Research Council of Canada to the first author and was conducted by the second author. Requests for reprints should be sent to Gary L. Wells, Department of Psychology, University of Alberta, Edmonton, Alberta, Canada T6G 2E9. critical difference rests not with the number of features processed or their distinctiveness, but instead with the match or mismatch between encoding operations and retrieval operations.

In general, we advocate an encoding-specificity framework (as in Tulving \& Thomson, 1973). Thus, retrieval is successful to the extent that the cognitive processes involved in encoding operations are similar to those involved in retrieval operations. Encoding specificity simply assumes that a witnessed event or episode can be best retrieved by using retrieval cues that are like those involved in the original episodic experience. Encoding specificity has a broad potential for explaining contextreinstatement effects (e.g., Bekerian \& Bowers, 1983; Fisher \& Craik, 1977), state-dependent learning (e.g., Bower, Montiero, \& Gilligan, 1978), and successful recall when recognition fails (e.g., Flexser \& Tulving, 1978).

We propose that trait-judgment-encoding operations with faces are dominated by holistic perceptual processing (see also Patterson \& Baddeley, 1977). There is much ambiguity in the literature regarding what is meant by holistic processing of faces. What we mean by holistic processing of faces is that there is topographical-type information being processed that might include relational cues among features as well as spatial-location cues. Holistic processing of faces may involve more than sim. ple topographical information (e.g., symmetry/asymmetry judgments), but it is characterized by its intact wholeness rather than its features in isolation. For example, if someone is asked to judge a face for honesty, it is unlikely that cognitive operations will involve isola- 
tion of features (e.g., a mental comparison of honest noses known in the past with the target nose). Instead, cognitive operations would be at a level at which the features are in holistic context (e.g., comparison of the face as a whole with a personal prototype of honest or dishonest faces). This is what is meant when we claim that trait judgments of faces foster holistic perceptual processes. By holistic we do not mean that a greater number of features have been processed (as in Winograd, 1978). Instead, we mean that between-feature information is being processed and thereby becomes a retrieval cue.

If holistic processing of faces is characterized by a topographical analogy, wherein there are betweenfeature comparisons regarding relative size, distance, or spatial location, then how is within-feature processing characterized? Within-feature processing also could be characterized in a topographical framework. Any given feature of the face has topographical information (e.g., distance between nostrils, distance between eyes, symmetry across lips) that aids feature retrieval. However, by holistic processing we mean that there is processing of interfeature topography rather than only intrafeature topography. By feature processing we mean processing of intrafeature topography only.

We propose that the recognition of faces, as a retrieval task, favors holistic-encoding operations because the relevant interfeature topographical cues to retrieval are preserved in the recognition task. This is consistent with previous speculation that face recognition is holistic rather than feature based (e.g., Baddeley, 1979; Ellis, Shepherd, \& Davies, 1975; Woodhead, Baddeley, \& Simmonds, 1979). We argue that it is the match between encoding and retrieval operations that is responsible for the superiority of trait encoding over feature encoding as measured by recognition tasks.

The concepts of interfeature processing and intrafeature processing help clarify our conception of holistic versus feature processing, However, it raises the question of what constitutes a feature. For example, if perceivers process face shape, have they processed one feature or have they processed multiple features (e.g., chin, cheekbone, and hair line)? In our analysis, face shape is a feature. We define a feature as an identifiable subset of the overall stimulus in question. Although any given feature could be construed as being composed of multiple features (e.g., an ear has lobes and other features), this does not constitute a serious drawback for our conceptualization. The critical aspect of our conceptualization has to do with the extent of interfeature versus intrafeature processing with respect to a given multifeatured stimulus. Tasks that require cognitive operations to be performed individually on one or more components of a complex stimulus involve less extensive interfeature processing than do tasks requiring overall analyses of the complex stimulus. In our conceptualization, it is inappropriate to think of holistic versus feature processing as a dichotomy; holistic processing simply is a convenient term to characterize a relatively extensive degree of interfeature processing. We will use the phrases holistic processing and feature processing herein to refer to the relative amounts of inter-and intrafeature processing wherein features are defined operationally in terms of identifiable components (e.g., nose, eyes, chin, hair, brows) of the complex stimulus (face).

A critical assumption in our conceptualization is that holistic processing at encoding is not necessarily better than feature processing at encoding in terms of subsequent retrieval. The critical determinant of retrieval success depends on the extent to which the retrieval cues at test are similar to those at encoding. Thus, a holistic encoding of faces might not favor an intrafeature-based retrieval task (e.g., the Identi-Kit) because the features would be out of context. In this case, the holistic-encoding operations would not match the level of operations at retrieval, namely, feature-based retrieval operations. Importantly, previous studies of trait versus feature encoding of faces have been restricted to full-face-recognition tasks.

If our interpretation is correct, it could be argued that a trait judgment of a face does not yield better memory or a more endurable trace than does making a physical-feature judgment of a face. Instead, the two encoding tasks simply favor different kinds of retrieval. In order to test this, we used a face-retrieval task that is feature based, namely, the Identi-kit. The Identi-kit is composed of a booklet that has separate facial features (hair, eyes, nose, brows, chin, mouth) that can be chosen by a person and put together with transparencies to make a face.

We predicted that, as in previous studies, having people make trait judgments of a face would better facilitate recognition accuracy than would having people make physical-feature judgments. However, we predicted the opposite for Identi-kit reconstruction accuracy: Namely, physical-feature judgments would produce better reconstructions than would trait judgments.

\section{METHOD}

\section{Subjects and Design}

Participants were 80 undergraduate psychology students who were meeting an introductory psychology course requirement. The subjects were assigned randomly to one of four conditions in a 2 (personality-trait judgment vs. physical-features judgment) $\times 2$ (recognition test vs. reconstruction task) betweensubjects factorial design.

\section{Materials}

All materials came from the Identi-kit package available from Smith \& Wesson; the kit consists of transparencies of facial features (i.e., noses, eyes,) with numbered variations of each feature. Specifically, there are 34 noses, 106 pairs of eyes, 177 hair styles, 9 types of moustaches, 37 lips, 26 chins, and 16 brows. The kit also consists of a booklet that contains numbered photographs corresponding to each transparency in the box. The stimulus shown to all subjects was a male face constructed from the Identi-kit (see Figure 1). Foil faces, used for subjects in the recognition conditions and in which the target face was embedded, were also constructed from the Identi-kit. The foils' similarity to the target was varied in pilot work to reach a level 
of task difficulty well below $100 \%$ but above chance. No foils shared exact features among themselves or with the target face.

\section{Procedure}

All participants were tested individually. They were all first given a cover story, to wit, "we are interested in what information people get from examining other people's faces," which was designed to be congruous with the subsequent task (i.e., personality-trait judgments or physical-features judgments) while not alerting the subject to the fact that a memory test (i.e., recognition or reconstruction) would follow. All subjects studied the stimulus face for $30 \mathrm{sec}$ during which time they rated the face according to their respective encoding task. Specifically, half of the participants were required to rate the face for 10 personality traits using a 7-point scale (i.e., to what extent does this person appear honest-dishonest?), whereas the other half rated the faces for 10 physical features (i.e., to what extent does this person have a long-short nose?). The 10 traits were honesty, intelligence, aggressiveness, kindness, generosity, imaginativeness, arrogance, friendliness, selfishness, and excitability, and the 10 physical-feature judgments were for eyes (close together-far apart), eyes (narrow-wide), nose (long-short), nose (broadnarrow), lips (thin-full), brows (thin-thick), cheekbones (prominent-indistinctive), jawline (angular-rounded), face shape (longshort), and face (narrow-wide).

The subjects then proceeded to the second part of the experiment, in which half from each encoding condition were selected randomly for testing in either the recognition condition or the reconstruction condition. After rating the stimulus face, the subjects in the recognition condition engaged in a 15 -min filler activity designed to mildly interfere with memory for the original face. This activity required the subjects to pair up eight photographed faces (four male faces and four female faces) "on the basis of whatever information they got from examining these faces." Following this filler activity, the subjects were then presented a lineup of six Identi-kit faces and were asked to "choose the face that you were shown originally." The stimulus face was always present, but its position among the five foil faces was varied randomly. Following their choices, the subjects were asked to indicate how certain they were that they had identified the correct face on a scale of 1 (totally uncertain) to 7 (totally certain).

In the reconstruction condition, after the subjects rated the face for either personality traits or physical features, they were presented with the Identi-kit and instructed to reconstruct the face. The reconstruction phase consisted of the subjects' choosing from the booklet each feature that they thought best resembled that of the original while the experimenter extracted the corresponding transparencies from the box, building the face in the subjects' full view. The order in which subjects encountered the features was determined largely by the Identi-kit booklet (hair, chin, eyes, lips, nose, brow, facial hair), although subjects were allowed to use any order and of ten skipped around. There were no time constraints, and the subjects were allowed to make as many changes as they wished until satisfied with the reconstruction. After finishing their reconstruction, the subjects were asked to examine the resultant face and to indicate how satisfied they were with their reconstruction on a scale of 1 (totally dissatisfied) to 7 (totally satisfied).

\section{Scoring of Data}

The subjects in the recognition conditions were assigned a score of either 1 or 0 , depending on whether or not they accurately identified the target face. Identi-kit reconstructions were scored in three ways by a separate sample of 80 subjects. First, 40 subjects were each shown all 40 Identi-kit reconstructions (i.e., 20 reconstructions from the trait-encoding condition and 20 from the feature-encoding condition) and were asked to choose between that reconstruction and another reconstruction (standard comparison) in terms of which better resembled the target face. This measure of reconstruction quality hereafter is called the standard-comparison measure. The standard-comparison face, the original target face, and a typical reconstruction from each encoding condition are shown in Figure $1 .{ }^{1}$ These judges were allowed to use any criterion they wished in terms of what constituted a better "resemblance." Each reconstruction thereby received a score from 0 to 40 , depending on how many of the judges thought it to be better than the standard in terms of its resemblance to the original. Five random orders were used for the 40 reconstructed faces.

Twenty other subjects scored each of the 40 reconstructed faces on a 7-point scale with endpoints marked "does not resemble the original" (1) and "closely resembles the original" (7). These subjects were asked to "make an 'overall' judgment on the basis of the entire face rather than specific features." Again, there were five random orders used for the 40 faces. This measure of reconstruction quality hereafter is referred to as the holistic rating measure.

Finally, 20 separate subjects judged each feature (hair, chin, eyes, lips, nose, brow, facial hair) for each face in comparison with the original on a 7 -point scale $(1=$ closely resembles; $7=$ does not resemble). For this judgment task, the features were presented separately (i.e., not as part of an intact face). Five random orders of features were used. These judges had to judge only 178 separate features, rather than 280 , because some of the 7 features were chosen more than once across the 40 reconstructed faces. Each face was assigned a score representing the mean rating of the 7 features. This measure of reconstruction quality hereafter is referred to as the individual feature rating. (A fourth scoring task was considered, wherein hits on exact features were scored. The frequency of exact hits on features was too low, however, to make it a sensitive measure. There were only four exact hits in the feature-encoding condition and two in the trait-encoding condition.)

\section{RESULTS}

The results on the recognition measure yielded a sig. nificant effect for encoding condition, wherein trait encoding was superior to feature encoding ( $55 \%$ accuracy vs. $25 \%$ accuracy, respectively, $Z=2.03, p<.05$ ). The three methods of scoring reconstructions were analyzed separately. The first method, comparing the reconstruction with the standard comparison in terms of which better resembled the original, yielded a significant effect favoring feature encoding $(m=19.2$ for trait encoding, $\mathrm{m}=28.6$ for feature encoding $, \mathrm{Z}=2.4, \mathrm{p}<.05$ ). A face from the feature-encoding condition and a face from the trait-encoding condition that yielded scores closest to their respective condition (on the standard-comparison measure) are shown in Figure 1. The second method, rating the reconstructions on a 7-point scale for "overall" resemblance rather than features, yielded a marginally sig. nificant effect favoring feature encoding $(m=1.6$ for trait encoding, $m=2.3$ for feature encoding, $Z=1.9$, $\mathrm{p}<.06$ ). The third method, rating the individual features on a 7-point scale, yielded significant differences favoring the feature-encoding condition $(m=1.3$ for trait-encoding, $m=22$ for feature encoding, $Z=2.0$, $p<.05)$.

There were no main effect or interaction differences between trait and feature conditions in terms of the sub- 

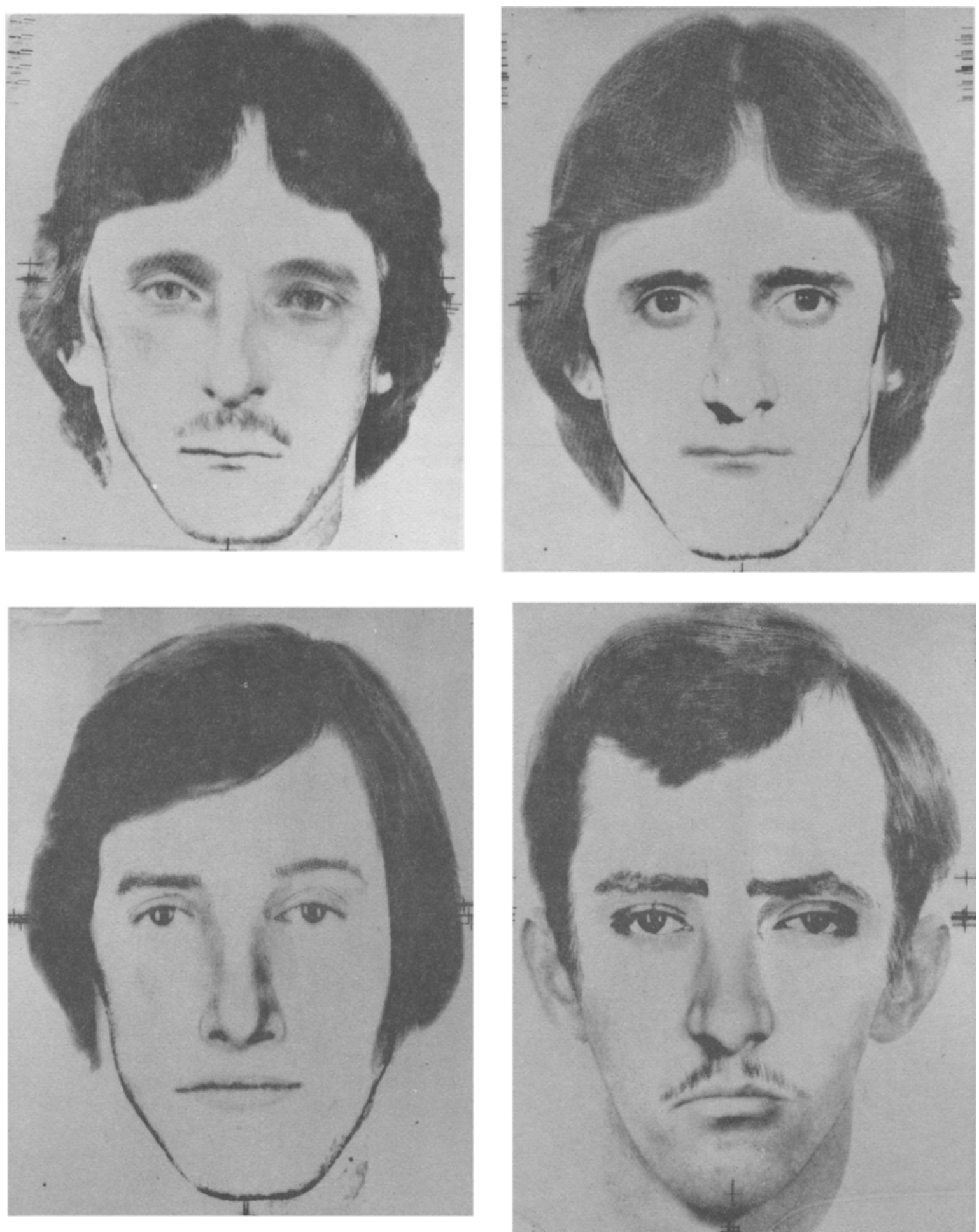

Figure 1. Target face, standard comparison face, and representative reconstructions from feature- and trait-encoding conditions. 
jects' mean certainty in their identification or satisfaction in reconstruction. However, a point-biserial correlation between the subjects' certainty in their identification and accuracy of identifications was positive and significant in the feature-encoding condition $(\mathrm{r}=+.43$, $p<.05$ ) and nonsignificant in the trait-encoding condition $(r=-.04, n . s$. $)$. The reverse of this pattern held for the correlation between subjects' satisfaction ratings and the quality of reconstruction scores for each of the measures of reconstruction quality. The correlations were $+.03,-.13,+.11(\mathrm{ps}>.05)$ for feature encoding using the standard-comparison measure, the holistic-rating measure, and the feature-rating method, respectively; rs $=+.41$ and +.46 , ps $<.05$ and +.26 , n.s., for the traitencoding conditions using the standard-comparison measure, the holistic-rating measure, and the feature-rating method, respectively.

Finally, the number of times that subjects in the reconstruction conditions changed features during their attempted reconstructions was compared in the two encoding conditions. Trait-encoding subjects changed more features $(m=3.25)$ than did feature-encoding subjects $(m=1.16)$, but the difference was not significant $(\mathrm{Z}=1.3, \mathrm{p}<.20)$.

\section{DISCUSSION}

Our conceptualization, based on an encoding-specificity framework, predicted a crossover interaction between encoding operations (trait judgments vs. feature judgments of faces) and retrieval operations (full-face recognition vs. feature-based recognition). The predicted interaction was robust; trait judgments were significantly better than feature judgments for full-face recognition, but feature judgments were significantly better than trait judgments for feature-based retrieval, using the Iden ti-kit.

These results have implications for the interpretation usually offered for the superiority of trait- over featureencoding operations for face recognition. Specifically, researchers have argued that trait-judgment encoding increases the number of distinctive features that are encoded compared with that involved in a feature-judgment task (e.g., Bower \& Karlin, 1972; Winograd, 1981; Yarmey, 1979, pp. 141-142). If this were true, however, trait-based encoding should have produced better featurebased reconstructions than did feature-based encoding; the opposite result obtained.

Alternatively, it is possible that the number-offeatures viewpoint can be maintained by invoking a conception of limited processing capacity (Kahneman, 1973). Specifically, it could be argued that trait encoders processed a greater number of features than did feature encoders but that each feature was processed less deeply (because of, e.g., temporal limits on overall processing). This explanation is difficult to reconcile with the fact that the retrieval task (Identi-kit) is exhaustive. That is, the retrieval task involved all facial features that characterized the stimulus face, thereby disallowing any bias in the feature-retrieval task toward features chosen in the feature-encoding conditions.

The results of this experiment are relevant to the debate regarding how to characterize holistic processing of faces and whether face recognition is holistic or feature based (e.g., Baddeley, 1979; Ellis et al., 1975; Woodhead et al., 1979). The current study suggests strongly that face recognition is characterized best by a holistic rather than by a feature-based process. The results of the current study also implicate holistic-encoding processes in the task of making trait judgments of faces.

The current paradigm, in which the subject is required either to recognize the target among distractors or to reconstruct the target using the Identi-kit, has potential for addressing the strategies people use to process faces under various conditions. Winograd (1976), for example, found that recognition accuracy following occupational judgments (e.g., "Does he look like a teacher?") was indistinguishable from trait judgments. This suggests to us that occupational judgments of faces follows a holistic process. Bower and Karlin (1974) found that judg. ments of gender were inferior to trait judgments on recognition accuracy. Our conceptualization suggests that this could be due to gender judgments' being feature based.

The current conceptualization also helps account for why researchers have found that the superiority of trait over feature-judgment tasks is not qualified by the perceiver's judgment (i.e., a "yes" or "no" response to a question of whether the nose is large or the person is friendly; see Winograd, 1976). According to the current conceptualization, the nature of the memory trace for faces is linked to perceptual processes (holistic vs. feature), which are not altered by the perceiver's resultant judgment.

An interesting ancillary finding was obtained with regard to the certainty with which recognition judgments were made and the satisfaction ratings that subjects gave to their reconstructions. Specifically, trait-encoding subjects were as certain in their false recognitions as they were in their accurate recognitions, whereas featureencoding subjects were less certain in their false recognitions than they were in their accurate recognitions. This finding, along with the fact that feature-encoding subjects performed more poorly than did trait-encoding subjects on the full-face-recognition task, suggests that feature encoders had additional information about the face that was not well tapped by those in the full-facerecognition condition. Complementing the recognitioncertainty finding is the reverse pattern that obtained for reconstruction-satisfaction correlations. Specifically, trait-encoding subjects reported greater satisfaction for good reconstructions than they did for poorer reconstructions, whereas feature-encoding subjects were equally satisfied with good versus poor reconstructions. This finding, along with the fact that trait-encoding subjects performed more poorly than did feature-encoding 
subjects on the reconstruction task, suggest that trait encoders had additional information about the face that was not well tapped by the reconstruction task. Thus, these results are consistent with our conceptualization that the feature versus trait $x$ full-face recognition versus feature reconstruction interaction is due to a match/ mismatch of encoding and retrieval tasks.

We admit that our conceptualization has some of the trappings of circularity. Specifically, we assume that extensive interfeature (holistic) processing is operative in making trait judgments of faces because the trait-encoding task aided whole-face recognition but not individual feature recognition, whereas feature-based encoding had the opposite effect. There is, however, no evidence regarding the interfeature- versus intrafeature-processing distinction independent of the encoding-retrieval interaction pattern obtained herein. Nevertheless, the conceptualization had some heuristic value: It allowed predictions that were consistent with data outcomes, and these predictions were not consistent with prior conceptions (e.g., the notion that trait judgments simply increase the number of distinctive features that are processed).

It is important to keep in mind that the Identi-kit construction task is not a recall test. It is, instead, a task that requires recognition of constituent features of the face. What would have happened had we used verbal recall of the faces for the memory test? Unlike the Identikit task, a verbal description is not strictly a feature-based form of retrieval, since it is common in verbal face recall to get global descriptors (e.g., "he looked mean"). However, verbal face recall is also not clearly holistic, since it is also common to get feature descriptors ("he had kind of a big nose"). This analysis is consistent with Wolfskiel and Brigham's (1983) recent finding that a trait task did not differ from a physical-judgment task on subjects' verbal memory descriptions of a target face. We suggest that this is because verbal recall of faces is not distinc. tively feature based or holistic based. Verbal descriptions commonly involve both processes (e.g., "he had a large nose and he looked mean").

The current conceptualization is relevant to findings that the quality of a person's Identi-kit reconstruction of a target face from memory is not related to the quality of the person's verbal description of the target face (e.g., Laughery \& Fowler, 1980) and that the quality of verbal descriptions of faces from memory does not relate to the person's ability to identify a face (e.g., Goldstein, Johnson, \& Chance, 1979). Our conceptualization suggests that there is some orthogonality in the types of retrieval cues involved in these face-memory tasks: Recognition of faces favors interfeature retrieval cues; reconstruction of faces from features favors intrafeature cues; verbal recall of faces favors neither inter- nor intrafeature cues.

We conceptualized the distinction between feature and holistic processing of faces in terms of the extent of intra- versus interfeature topographical processing. We believe this is an improvement over prior, less explicit accounts of the holistic versus feature distinction in the literature on face processing. By defining what we meant as feature versus holistic processing, the language of feature versus holistic served our purposes. However, we question whether the feature versus holistic distinction might better be replaced by more explicit hypotheses of what is being processed. In the generic case, any stimulus (e.g., faces, scenes, words) can be construed as having components (or features or units); the extent to which intracomponent information (e.g., symmetry, shape) rather than intercomponent information (e.g., distances between components, relative sizes of components) is processed has important implications for the representation of encodings and the kinds of retrieval operations that will be successful. A language of processing that specifies components and uses an intracomponent versus intercomponent distinction may prove more useful than has the global holistic versus feature distinction.

\section{REFERENCES}

Baddeley, A. D. (1979). Applied cognitive and cognitive applied psychology: The case of face recognition. In L. G. Nilsson (Ed.), Perspectives on memory research. Hillsdale, NJ: Erlbaum.

Benerian, D. A., \& Bowers, J. M. (1983). Eyewitness testimony: Were we misled? Journal of Experimental Psychology: Learning, Memory, and Cognition, 9, 139-145.

Bower, G. H., \& KarLin, M. B. (1974). Depth of processing pictures of faces and recognition memory. Journal of Experimental Psychology, 103, 751-757.

Bower, G. H., Monteiro, K. P., \& Gilligan, S. G. (1978). Emotional mood as a context in learning and recall. Journal of Verbal Learning and Verbal Behavior, 17, 573-585.

Craik, F. I. M., \& LockhaRT, R. S. (1972). Levels of processing: A framework for memory research. Journal of Verbal Learning and Verbal Behavior, 11, 671-684.

Ellis, H. D., She phard, J. W., \& Davies, G. M. (1975). An investigation of the use of the Photofit technique for recalling faces. British Journal of Psychology, 66, 29-37.

Fisher, R. P., \& Craik, F. I. M. (1977). The interaction between encoding and retrieval operations in cued recall. Journal of $E x$ perimental Psychology: Human Learning and Memory, 3, $701-711$.

Flexser, A. J., \& Tulving, E. (1978). Retrieval independence in recognition and recall. Psychological Review, 85, 153-171.

Goldste in, A. G., Johnson, K. S., \& Chance, J. (1979). Does fluency of face description imply superior face recognition? Bulletin of the Psychonomic Society, 13, 15-18.

Kahneman, D. (1973). Attention and effort. Englewood Cliffs, NJ: Erlbaum.

Laughery, K. R., \& Fowlen, R. F. (1980). Sketch artist and Identi-kit procedures for recalling faces. Journal of Applied Psychology, 65, 307-316.

Mueller, J. H., Cahlomusto, M., \& Goldstein, A. G. (1978). Orienting task and study time in facial recognition. Bulletin of the Psychonomic Society, 11, 313-316.

Patterson, K. E., \& Baddeley, A. D. (1977). When face recognition fails. Journal of Experimental Psychology: Human Learning and Memory, 3, 406-417.

Stranad, B. N., \& Mueller, J. H. (1977). Levels of processing in facial recognition memory. Bulletin of the Psychonomic Society, 9, 17-18. 
Tulving, E., \& Thomson, D. M. (1973). Encoding specificity and retrieval processes in episodic memory. Psychological Review, 80, 352-373.

Warrington, E. K., \& Ackroyd, C. (1975). The effect of orienting tasks on recognition memory. Memory \& Cognition, 3 , 140-142.

Winograd, E. (1976). Recognition memory for faces following nine different judgments. Bulletin of the Psychonomic Society, 8, 419-421.

WinOGRAD, E. (1978). Encoding operations which facilitate memory across the life span. In M. M. Gruneberg, P. E. Morris, \& R. N. Sykes (Eds.), Practical aspects of memory. London: Academic Press.

WiNOGRAD, E. (1981). Elaboration and distinctiveness in memory for faces. Journal of Experimental Psychology: Human Learning and Memory, 7, 181-190.

Wolfskiel, M. P., \& Brigham, J. C. (1983). Prior description and face recognition. Unpublished manuscript, Florida State University, Department of Psychology, Tallahassee, FL.
Woodhead, M. M., Baddeley, A. D., \& Simmonds, D. C. V. (1979). On training people to recognize faces. Ergonomics, 22, 333-343.

YARMEY, A. D. (1979). The psychology of eyewitness testimony. New York: Free Press.

NOTE

1. The "typical" reconstructions in Figure 1 were selected on the basis of their proximity to the mean for their respective encoding conditions. The face reconstructed from the featureencoding condition yielded 30 of 40 possible choices from the judges (cell mean $=28.6$ ). The face reconstructed from the traitencoding condition yielded 19 of a possible 40 choices (cell mean $=19.2$ )

(Manuscript received December 1, 1983 ; revision accepted for publication April 23, 1984.) 